\title{
Revamp the MOC
}

\section{There are few things that psychiatrists}

have come to despise more than the

American Board of Psychiatry and

Neurology (ABPN) Maintenance of

Certification (MOC) program. It has

become a professional boondoggle

for psychiatric practitioners.

The program needs an overhaul and simplification. There are better, more efficient, cost-effective ways to ensure psychiatric physicians' ongoing clinical competence after they complete their residency training. Technological advances can also facilitate a more valid assessment of competence without having to jump through more and more hoops between recertifications every 10 years.

I passed the boards long before the MOC was created. For 20 years, I also served as a senior examiner for the oral boards, where clinical competency was rigorously assessed by direct observations of psychiatrists examining and establishing rapport with patients and formulating the data into a differential diagnosis, treatment plan, and prognosis. It is noteworthy that psychiatrists who sat for the oral boards had already passed a written exam that tested their cognitive knowledge. Yet approximately one-third of the candidates failed the live oral exam, which clearly implies that passing a written exam is necessary but not sufficient to establish clinical competence, which is the primary purpose of board certification. It was an unfortunate decision to discontinue the face-to-face oral board exam, which is so vital for psychiatry, and to replace it with a written exam and a barrage of timeconsuming activities to document lifelong learning and self-assessment, but not genuine clinical competence. The MOC has been MOCkingly referred to as a major pain in the neck for practically all psychiatrists who were not grandfathered with lifetime certification, as was the case in the first 60 years of the ABPN.

\section{Benefits of the patient-based oral exam}

Let's face it: Passing a patient-based oral exam was the ideal mechanism to establish that a psychiatric physician deserved to be a diplomate of the ABPN. During the oral exam, the candidate's skills were observed from the minute he/she met the patient. The candidate was then observed as he/ she systematically explored a wide range of past and current psychiatric symptoms; reviewed the patient's developmental, medical, family, and social histories; and conducted a competent mental status exam while demonstrating an empathic stance, responding to the patient's often subtle verbal and nonverbal cues, establishing rapport, and providing psychoeducation before concluding the interview. All these essential components of a psychiatric exam were observed in a compact 30-minute tour de force of clinical skills, communication, and cognitive acumen. This was followed by another 30 minutes of organizing and presenting the clinical

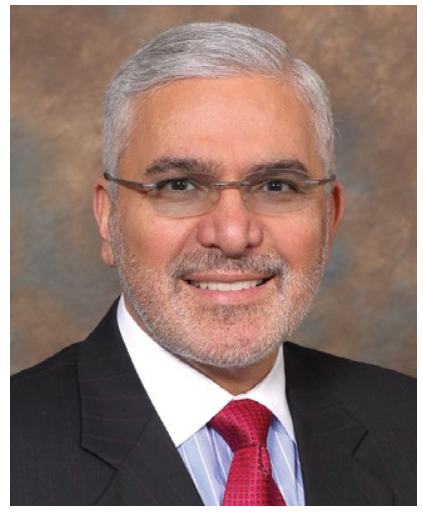

Henry A. Nasrallah, MD Editor-in-Chief

doi: $10.12788 /$ cp. 0038

Technological advances can be used to create a more efficient and cost-effective clinical assessment of psychiatric competence
To comment on this editorial or other topics of interest:

henry.nasrallah

@currentpsychiatry.com 


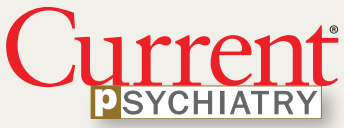

Editorial Staff

EDITOR Jeff Bauer

SENIOR EDITOR Sathya Achia Abraham

ASSISTANT EDITOR Jason Orszt

WEB ASSISTANTS

Tyler Mundhenk, Kathryn Wighton

Art \& Production Staff

CREATIVE DIRECTOR Louise Koenig

ART DIRECTOR Pat Fopma

DIRECTOR, JOURNAL MANUFACTURING Michael Wendt

PRODUCTION MANAGER Donna Pituras

Publishing Staff

PUBLISHER Sharon Finch

DIRECTOR EBUSINESS DEVELOPMENT

Alison Paton

SENIOR DIRECTOR OF SALES

Tim LaPella

Editor-in-Chief Emeritus

James Randolph Hillard, MD

Frontline Medical Communications

VP, SALES Mike Guire

VP, DIGITAL CONTENT \& STRATEGY Amy Pfeiffer

PRESIDENT, CUSTOM SOLUTIONS JoAnn Wahl

VP, HUMAN RESOURCES \& FACILITY OPERATIONS Carolyn Caccavelli CIRCULATION DIRECTOR Jared Sonners DIRECTOR, CUSTOM PROGRAMS Patrick Finnegan

In affiliation with Global Academy for Medical Education, LLC

PRESIDENT David J. Small, MBA

\section{FRONTLLNE MLedge}

7 Century Drive, Suite 302

Parsippany, NJ 07054

Tel: (973) 206-3434

Fax: (973) 206-9378

www.frontlinemedcom.com

Subscription Inquiries:

subscriptions@mdedge.com

Published through an educational partnership with

\section{l(C CINCINNATI}

Current Psychiatry

8 September 2020 data to 2 or 3 colleagues/examiners, in a coherent fashion, connecting all the dots, formulating the case, presenting a meaningful differential diagnosis, and suggesting a rational array of potential treatment options across the biopsychosocial continuum. To top it off, the candidate had to respond effectively, in an evidence-based manner, to a series of questions related to the disease state, its treatment, adverse effects, and prognosis.

It was a joy to watch many colleagues navigate this clinical examination with skill and competence, without crumbling under the pressure of the examiners' scrutiny. There were some who passed with flying colors, and others who passed despite having a forgivable minor gap here and there because of their overall strong performance. Finally, there were those who stumbled in several components across data collection, doctor-patient interactions, synthesis of the clinical findings, or treatment recommendations. These candidates inevitably received a failing grade by a consensus of 3 examiners. That they failed to demonstrate clinical competence despite having passed the required written exams a year earlier proved that the true competency of a psychiatrist cannot be judged solely by passing a written test but requires a clinical examination of a live patient.

The oral exams represented an unimpeachable evaluation of clinical competence. The examiners often spoke of how they would feel confident and comfortable with referring a family member to those who successfully passed this rigorous, authentic exam on real patients. It was justifiable to give lifetime certification to those who passed the oral exam. Those permanently certified psychiatrists maintained their lifelong learning by having an unrestricted state medical license, which is contingent on acquiring 50 category 1 continuing medical education (CME) credits annually. Why not restore lifelong certification for those who pass both a written and oral exam, as long as they maintain a valid medical license?

According to the ABPN 2019 Annual Report, ${ }^{1}$ 31,514 psychiatrists have received lifetime certification, of whom an estimated 9,547 were still clinically active in 2019. This is the "grandfathered" cohort of psychiatrists to which I belong. I was tested on neurologic patients, not just psychiatric patients, a tribute to the strong bridge that existed between these sister brain specialties. As of 2019, of the 33,277 psychiatrists who received a time-limited certification, 29,343 were still clinically active, an attrition rate of $12 \%$ over the past 25 years. This includes psychiatrists who found the MOC too onerous to complete, or are in private practice where MOC is not a vital requirement. However, these days most psychiatrists are obligated to be recertified because so many entities require it. This includes hiring institutions, government agencies (Medicare/Medicaid), health insurance companies, hospital medical staff for privileging and credentialing, and various regulatory boards, such as The Joint Commission, the Accreditation Council for Graduate Medical Education, and academic medical centers. Because most psychiatrists are involved with at least one of these entities, 29,343 have no choice but to perform all the requirements of the MOC, with its countless hours, numerous documentations, and many fees, to remain certified by the ABPN. Notably absent is an alternative mechanism for a certification process that is widely accepted by all agencies and institutions. Psychiatrists are actively seeking alternatives. 
The ABPN, long regarded as an esteemed nonprofit organization, has been accused of being a monopoly. Some angry psychiatrists have filed a class action lawsuit to demand other board certification methods. Some have gone to the media to complain about the American Board of Medical Specialties (of which the ABPN is a member board), accusing both of unfair regulations or of raking in substantial profits to support excessively compensated executives. Perception often trumps reality, so no matter how vigorously the ABPN defends itself, its procedures, or its MOC requirements, its customerspsychiatric physicians-feel oppressed or exploited.

\section{How the MOC can be improved}

So what can be done to improve the MOC? The need for recertification is arguably necessary to document clinical competency over an approximately 40-year psychiatric career following residency. I conducted a brief survey of Current Psychiatry readers. Of the 319 respondents, $86.5 \%$ recommended abolishing the MOC, while $13.5 \%$ said it should remain or were unsure. In a follow-up question, $60 \%$ agreed that the American Psychiatric Association (APA) should establish a Council on Board Certification, and 33\% agreed that the MOC should only be a clinical vignette-based written exam every 10 years, along with an unrestricted or active state medical license.

Significant advances in remote communication technology should be harnessed by the ABPN (or the APA, if it decides to conduct its own board certification) to restore the old model at a fraction of the cost. The oral exams have been replaced by a written exam that is not an accurate reflection or documentation of clinical competence. The traditional oral exam (after passing a written exam) was a magnificent but costly feat of massive logistical complexity, with $>1,000$ candidates and examiners traveling to a city where the ABPN arranged for several hospitals to shut down their clinics for 2 full days to use their clinical offices for the oral exams. Multiple teams examined the candidates twice on the same day: once with a live patient, and again with a video of a real patient. The examiners filled out scoring cards after observing the candidates conduct the live interview or discussing the video. A consensus grade of pass or fail was documented. At the end of the 2 days, examiners and candidates boarded buses to the airport. It was a highly expensive process (exam fees + airfare + hotel + food). Twice a year, the examiners generously donated their time to the ABPN without compensation, as a token of love for and service to the profession.

That initial certification of a written exam, followed by an oral exam, validated the competence of a psychiatrist both cognitively and clinically. The lifetime certification was truly earned. The same model can now be replicated virtually via videoconferencing at a far lower cost to the ABPN, the candidates, and the examiners. The MOC 10-year recertification can be reduced to a written exam with clinical vignettes and an unrestricted license to practice medicine in any state, which implies that the psychiatrist has received the $50 \mathrm{CME}$ annual credits to renew the license. The rest of the bells and whistles can be strongly recommended but not required. The cost in time and money to both the ABPN and the candidates can be significantly reduced, but more importantly, the clinical competence will be validated at baseline with virtual oral boards after passing the written exam (formerly labeled as part I, preceding the part II oral boards).
Significant advances in remote communications technology can be used to restore the old model of the MOC at a fraction of the cost 
Psychiatrists should be able to focus on treating patients, instead of being burdened by the many time-consuming requirements of the $\mathrm{MOC}$
The traditional board certification model of the past should be resurrected via videoconferencing and offered as an option to the candidates who prefer it to the current MOC. The MOC can then be simplified to lifetime certification or to only a written exam with clinical vignettes every 10 years to ensure that psychiatrists continue to incorporate relevant clinical and treatment advances in their practice. The KISS principle (keep it simple, stupid) worked very well for many generations of psychiatrists in the past, and will work again going forward if offered as an option. Psychiatrists can then focus on treating patients instead of being burdened by the many timeconsuming requirements and hoops of the current MOC.

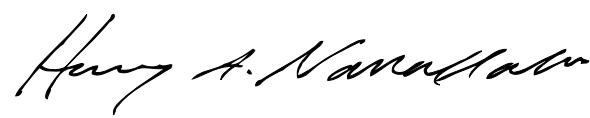

Henry A. Nasrallah, MD

Editor-in-Chief

\section{Reference}

1. American Board of Psychiatry and Neurology. 2019 Annual Report. https://www.abpn.com/wpcontent/uploads/2020/05/ABPN_2019_Annual_ Report.pdf. Accessed August 14, 2020. 